\title{
Linear Weingarten factorable surfaces in isotropic spaces
}

\author{
Muhittin Evren Aydin and Alper Osman Ogrenmis
}

\begin{abstract}
In this paper, we deal with a certain factorable surface in the isotropic 3 -space satisfying $a K+b H=c$, where $K$ is the relative curvature, $H$ the isotropic mean curvature and $a, b, c \in \mathbb{R}$. We obtain a complete classification for such surfaces. As a further study, we prove that a certain graph surface with $K=H^{2}$ is either a non-isotropic plane or a parabolic sphere.
\end{abstract}

Mathematics Subject Classification (2010): 53A35, 53A40, 53B25.

Keywords: Isotropic space, factorable surface, Weingarten surface, Euler inequality.

\section{Introduction}

Let $M^{2}$ be a regular surface of a Euclidean 3 -space $\mathbb{R}^{3}$ and $\kappa_{1}, \kappa_{2}$ its principal curvatures. Then $M^{2}$ is called a Weingarten surface if the following non-trivial functional relation occurs:

$$
\phi\left(\kappa_{1}, \kappa_{2}\right)=0
$$

for a smooth function $\phi$ of two variables. (1.1) immediately yields

$$
\delta(K, H)=0,
$$

where $K$ and $H$ are respectively the Gaussian and mean curvatures of $M^{2}$. (1.2) is equivalent to the vanishing of the corresponding Jacobian determinant, i.e. $|\partial(K, H) / \partial(u, v)|=0$ for a coordinate pair $(u, v)$ on $M^{2}$. If $M^{2}$ is a surface that satisfies

$$
a H+b K=c, a, b, c \in \mathbb{R}, \quad(a, b, c) \neq(0,0,0),
$$

then it is called a linear Weingarten surface ( $L W$-surface). If $a=0$ or $b=0$ in (1.3), then the LW-surfaces reduce to the surfaces with constant curvature. Such surfaces have been extensively studied, see [7, 8], [13]-[17], [30].

On the other hand, a surface in $\mathbb{R}^{3}$ that is the graph of the function $z(x, y)=$ $f(x) g(y)$ is said to be factorable or homothetical. In various ambient spaces, these 
surfaces have been desribed in terms of their curvatures and Laplace operator in $[4,9,10,12,18,19,28,29]$. As distinct from the Euclidean case, a graph surface in the isotropic space $\mathbb{I}^{3}$ is said to be factorable if it is graph of either $z(x, y)=$ $f(x) g(y)$ or $x(y, z)=f(y) g(z)$. We call them the factorable surface of type 1 and type 2 , respectively. Note that the factorable surface of one type cannot be carried into that of another type by the isometries of $\mathbb{I}^{3}$. These surfaces of both type in $\mathbb{I}^{3}$ with $K, H=$ const. were obtained in [1]-[3].

The main purpose of this paper is to obtain LW-factorable surfaces of type 1 in $\mathbb{I}^{3}$. As a further study, we classify the graph surfaces of the function $z=z(x, y)$ in $\mathbb{I}^{3}$ with $K=H^{2}$.

\section{Preliminaries}

For general references of the isotropic geometry, see [5], [23]-[27]. The isotropic 3space $\mathbb{I}^{3}$ is a Cayley-Klein space defined from a 3 -dimensional projective space $P\left(\mathbb{R}^{3}\right)$ with the absolute figure $\left(\omega, f_{1}, f_{2}\right)$, where $\omega$ is a plane in $P\left(\mathbb{R}^{3}\right)$ and $f_{1}, f_{2}$ are two complex-conjugate straight lines in $\omega$. The homogeneous coordinates in $P\left(\mathbb{R}^{3}\right)$ are introduced in such a way that the absolute plane $\omega$ is given by $X_{0}=0$ and the absolute lines $f_{1}, f_{2}$ by $X_{0}=X_{1}+i X_{2}=0, X_{0}=X_{1}-i X_{2}=0$. The intersection point $F(0: 0: 0: 1)$ of these two lines is called the absolute point. The affine coordinates in $P\left(\mathbb{R}^{3}\right)$ are given by $x_{1}=\frac{X_{1}}{X_{0}}, x_{2}=\frac{X_{2}}{X_{0}}, x_{3}=\frac{X_{3}}{X_{0}}$. The group of motions of $\mathbb{I}^{3}$ is defined by

$$
\left(x_{1}, x_{2}, x_{3}\right) \longmapsto\left(x_{1}^{\prime}, x_{2}^{\prime}, x_{3}^{\prime}\right):\left\{\begin{array}{l}
x_{1}^{\prime}=a_{1}+x_{1} \cos \phi-x_{2} \sin \phi \\
x_{2}^{\prime}=a_{2}+x_{1} \sin \phi+x_{2} \cos \phi \\
x_{3}^{\prime}=a_{3}+a_{4} x_{1}+a_{5} x_{2}+x_{3},
\end{array}\right.
$$

where $a_{1}, \ldots, a_{5}, \phi \in \mathbb{R}$.

Consider the points $x=\left(x_{1}, x_{2}, x_{3}\right)$ and $y=\left(y_{1}, y_{2}, y_{3}\right)$. The isotropic distance $d_{\mathbb{I}}(x, y)$ of two points $x$ and $y$ is defined as

$$
d_{\mathbb{I}}(x, y)=\left(y_{1}-x_{1}\right)^{2}+\left(y_{2}-x_{2}\right)^{2} .
$$

The lines in $x_{3}$-direction are called isotropic lines. The plane containing an isotropic line is called an isotropic plane. Other planes are non-isotropic.

Let $M^{2}$ be a graph surface immersed in $\mathbb{I}^{3}$ corresponding to a real-valued smooth function $z=z(x, y)$ on an open domain $D \subseteq \mathbb{R}^{2}$. Then it is parameterized as follows:

$$
r: D \subseteq \mathbb{R}^{2} \longrightarrow \mathbb{I}^{3},(x, y) \longmapsto(x, y, z(x, y)) .
$$

It follows from (2.1) that $M^{2}$ is an admissible (i.e. without isotropic tangent planes). The metric on $M^{2}$ induced from $\mathbb{I}^{3}$ is given by $g_{*}=d x^{2}+d y^{2}$. This implies that $M^{2}$ is always flat with respect to the induced metric $g_{*}$ and thus its Laplacian is of the form $\triangle=\frac{\partial^{2}}{\partial x^{2}}+\frac{\partial^{2}}{\partial y^{2}}$. The relative (or isotropic Gaussian) curvature $K$ and the isotropic mean curvature $H$ of $M^{2}$ are defined by

$$
K=z_{x x} z_{y y}-\left(z_{x y}\right)^{2}
$$


and

$$
H=\frac{\triangle z}{2}=\frac{z_{x x}+z_{y y}}{2} .
$$

$M^{2}$ is called isotropic minimal (resp. isotropic flat) if $H$ (resp. $K$ ) vanishes.

\section{LW-factorable surfaces of type 1}

Let $M^{2}$ be a factorable surface of type 1 in $\mathbb{I}^{3}$, i.e., the graph of $z(x, y)=f(x) g(y)$. By $(2.2)$ and $(2.3)$, we get

$$
K=\left(f^{\prime \prime} f\right)\left(g^{\prime \prime} g\right)-\left(f^{\prime}\right)^{2}\left(g^{\prime}\right)^{2}
$$

and

$$
2 H=f^{\prime \prime} g+f g^{\prime \prime},
$$

where $f^{\prime}=\frac{d f}{d x}$ and $g^{\prime}=\frac{d g}{d y}$, etc. We mainly aim to classify the LW-factorable surfaces of type 1 in $\mathbb{I}^{3}$. For this, let $M^{2}$ satisfy the relation (1.3). Since at least one of $a, b$ and $c$ is nonzero in (1.3), without loss of generality, we may assume $b \neq 0$. By dividing both sides of (1.3) with $b$ and putting $\frac{a}{b}=2 m_{0}$ and $\frac{c}{b}=n_{0}$, we write

$$
2 m_{0} H+K=n_{0}, m_{0}, n_{0} \in \mathbb{R} .
$$

If $m_{0}=0, M^{2}$ turns to be a factorable surface of type 1 in $\mathbb{I}^{3}$ with $K=$ const. however such surfaces were already provided in [1]. In our framework, it is meaningful to take $m_{0} \neq 0$. By $(3.1)-(3.3)$, we get

$$
\left(f^{\prime \prime} f\right)\left(g^{\prime \prime} g\right)-\left(f^{\prime}\right)^{2}\left(g^{\prime}\right)^{2}+m_{0}\left(f^{\prime \prime} g+f g^{\prime \prime}\right)=n_{0} .
$$

We have to distinguish several cases in order to solve (3.4). Remark that the roles of $f$ and $g$ are symmetric, so discussing on the cases based on $f$ shall be sufficient. From now on, we use the notation $c_{i}$ to denote nonzero constants and $d_{i}$ to denote some constants, $i=1,2,3, \ldots$

Case 1. $f(x)=f_{0} \in \mathbb{R}-\{0\}$. By (3.4), we find

$$
g(y)=\frac{n_{0}}{2 f_{0} m_{0}} y^{2}+d_{1} y+d_{2} .
$$

Thereby, $M^{2}$ is isotropic flat factorable surface of type 1 with $H=\frac{n_{0}}{2 m_{0}}$.

Case 2. $f$ is a linear function, i.e. $f(x)=c_{1} x+d_{3}$. It follows from (3.4) that

$$
m_{0} d_{3} g^{\prime \prime}-c_{1}^{2}\left(g^{\prime}\right)^{2}+\left(m_{0} c_{1} g^{\prime \prime}\right) x=n_{0} .
$$

(3.6) implies that $g^{\prime \prime}=0$, namely $g(y)=c_{2} y+d_{4}$. In this case, $M^{2}$ is isotropic minimal factorable surface of type 1 with $K=-\left(c_{1} c_{2}\right)^{2}$.

Case 3. $f$ is a non-linear function. From the symmetry, $g$ is also a non-linear function. By dividing (3.4) with the product $f f^{\prime \prime}$, we get

$$
g^{\prime \prime} g-\frac{\left(f^{\prime}\right)^{2}}{f f^{\prime \prime}}\left(g^{\prime}\right)^{2}+m_{0} \frac{g}{f}+m_{0} \frac{g^{\prime \prime}}{f^{\prime \prime}}=\frac{n_{0}}{f f^{\prime \prime}} .
$$


By taking partial derivative (3.7) with respect to $y$ and then dividing with $g^{\prime} g^{\prime \prime}$, we deduce

$$
1+\frac{g g^{\prime \prime \prime}}{g^{\prime} g^{\prime \prime}}-2 \frac{\left(f^{\prime}\right)^{2}}{f f^{\prime \prime}}+\left(\frac{m_{0}}{f}\right) \frac{1}{g^{\prime \prime}}+\left(\frac{m_{0}}{f^{\prime \prime}}\right) \frac{g^{\prime \prime \prime}}{g^{\prime} g^{\prime \prime}}=0
$$

We have two cases:

Case 3.1. $g^{\prime \prime \prime}=0$, i.e.

$$
g(y)=c_{3} y^{2}+d_{5} y+d_{6} .
$$

Up to suitable translations of $y$, we may assume $d_{5}=d_{6}=0$. Then (3.8) reduces to

$$
1-2 \frac{\left(f^{\prime}\right)^{2}}{f f^{\prime \prime}}+\left(\frac{m_{0}}{2 c_{3}}\right) \frac{1}{f}=0
$$

(3.10) can be rewritten as

$$
\left(\frac{m_{0}}{2 c_{3}}+f\right) f^{\prime \prime}-2\left(f^{\prime}\right)^{2}=0
$$

After solving (3.11), we find

$$
f(x)=-\left(\frac{1}{c_{4} x+d_{7}}+\frac{m_{0}}{2 c_{3}}\right) .
$$

Considering (3.9) and (3.12) into (3.4) gives the contradiction

$$
x=-\frac{1}{c_{4}}\left(\frac{2 m_{0} c_{3}}{n_{0}+m_{0}^{2}}+d_{7}\right)
$$

due to the fact that $x$ is an independent variable.

Case 3.2. $g^{\prime \prime \prime} \neq 0$. By taking partial derivatives of (3.8) with respect to $x$ and $y$, we conclude

$$
\left(\frac{f^{\prime}}{f^{2}}\right) \frac{g^{\prime \prime \prime}}{\left(g^{\prime \prime}\right)^{2}}-\frac{f^{\prime \prime \prime}}{\left(f^{\prime \prime}\right)^{2}}\left(\frac{g^{\prime \prime \prime}}{g^{\prime} g^{\prime \prime}}\right)^{\prime}=0
$$

Due to $f^{\prime} g^{\prime \prime \prime} \neq 0$, neither $f^{\prime \prime \prime}$ nor $\left(\frac{g^{\prime \prime \prime}}{g^{\prime} g^{\prime \prime}}\right)^{\prime}$ can vanish in (3.13). Then $(3.13)$ can be rewritten as

$$
\frac{f^{\prime}\left(f^{\prime \prime}\right)^{2}}{f^{2} f^{\prime \prime \prime}}=\frac{\left(g^{\prime \prime}\right)^{2}}{g^{\prime \prime \prime}}\left(\frac{g^{\prime \prime \prime}}{g^{\prime} g^{\prime \prime}}\right)^{\prime}
$$

Since the left side of (3.14) is a function of $x$, however the right side is a function of $y$. Then both sides have to be equal a nonzero constant, namely

$$
\frac{f^{\prime}\left(f^{\prime \prime}\right)^{2}}{f^{2} f^{\prime \prime \prime}}=c_{5}=\frac{\left(g^{\prime \prime}\right)^{2}}{g^{\prime \prime \prime}}\left(\frac{g^{\prime \prime \prime}}{g^{\prime} g^{\prime \prime}}\right)^{\prime}
$$

From the left side of $(3.15)$, we write

$$
\frac{f^{\prime \prime \prime}}{\left(f^{\prime \prime}\right)^{2}}=\frac{1}{c_{5}} \frac{f^{\prime}}{f^{2}}
$$

or, by taking once integral with respect to $x$,

$$
f^{\prime \prime}=\frac{c_{5} f}{c_{5} d_{8} f+1}
$$


Likewise, by the right side of (3.15), we deduce

$$
\frac{g^{\prime \prime \prime}}{g^{\prime} g^{\prime \prime}}=\frac{-c_{5}}{g^{\prime \prime}}+d_{9}
$$

Substituting (3.17) and (3.18) into (3.8) yields

$$
\begin{aligned}
& 1+\left(m_{0} d_{8}+g\right) d_{9}-\frac{c_{5}\left(m_{0} d_{8}+g\right)}{g^{\prime \prime}}+\frac{m_{0} d_{9}}{c_{5} f}- \\
& -\frac{2\left(c_{5} d_{8} f+1\right)\left(f^{\prime}\right)^{2}}{c_{5} f^{2}}=0 .
\end{aligned}
$$

Taking partial derivative of (3.19) with respect to $y$ and considering (3.18) leads to

$$
g^{\prime \prime}=-c_{5}\left(g+m_{0} d_{8}\right) .
$$

After substituting (3.20) into (3.19), we conclude

$$
2+m_{0} d_{8} d_{9}+d_{9} g+\frac{m_{0} d_{9}}{c_{5} f}-\frac{2\left(f^{\prime}\right)^{2}}{f f^{\prime \prime}}=0,
$$

which yields $d_{9}=0$ and $f f^{\prime \prime}=\left(f^{\prime}\right)^{2}$. Solving this one gives $f(x)=c_{6} \exp \left(c_{7} x\right)$. By putting this in (3.4) we derive the polynomial equation on $(f)$ :

$$
c_{7}^{2}\left[g g^{\prime \prime}-\left(g^{\prime}\right)^{2}\right] f^{2}+m_{0}\left(c_{7}^{2} g+g^{\prime \prime}\right) f-n_{0}=0,
$$

which implies that the coefficients must be zero; namely $n_{0}=0$,

$$
g g^{\prime \prime}-\left(g^{\prime}\right)^{2}=0 \text { and } c_{7}^{2} g+g^{\prime \prime}=0 .
$$

(3.21) leads to the contradiction $c_{7}^{2} g^{2}+\left(g^{\prime}\right)^{2}=0$ and therefore we have proved the following:

Theorem 3.1. Let $M^{2}$ be a $L W$-factorable surface of type 1 which is the graph of $z(x, y)=f(x) g(y)$ in $\mathbb{I}^{3}$. Then we have either

(A) $f(x)=f_{0} \in \mathbb{R}-\{0\}, g(y)=c_{6} y^{2}+d_{10} y+d_{11}$;

(B) or $z(x, y)=\left(c_{7} x+d_{12}\right)\left(c_{8} y+d_{13}\right)$.

\section{Graph surfaces with $K=H^{2}$}

Let $M^{2}$ be a surface of the Euclidean 3-space $\mathbb{R}^{3}$. The Euler inequality for $M^{2}$ including the Gaussian and mean curvature follows

$$
K \leq H^{2}
$$

The equality sign of (4.1) holds on $M^{2}$ if and only if it is totally umbilical, i.e. a part of a plane or a two sphere in $\mathbb{E}^{3}$. For more generalizations, see [6, 11], [20]-[22]. Now we are interested in the factorable surfaces of type 1 in $\mathbb{I}^{3}$ satisfying $K=H^{2}$. For this, let us reconsider (3.1) and (3.2). If $K=H^{2}$, then

$$
\left(f^{\prime \prime} g-f g^{\prime \prime}\right)^{2}+4\left(f^{\prime} g^{\prime}\right)^{2}=0 .
$$

(4.2) immediately implies that

$$
f^{\prime \prime} g-f g^{\prime \prime}=0 \text { and } f^{\prime} g^{\prime}=0 .
$$


By (4.3) we conclude that either $f=$ const. and $g(y)=c_{1} y+d_{1}$ or $g=$ const. and $f(x)=c_{2} x+d_{2}$, which yields the following result:

Proposition 4.1. The factorable surfaces of type 1 in $\mathbb{I}^{3}$ satisfying $K=H^{2}$ are only non-isotropic planes.

As a generalization, we are able to investigate the graph surfaces of type 1 in $\mathbb{I}^{3}$ satisfying $K=H^{2}$. More precisely, let $M^{2}$ be a graph surface of $z=z(x, y)$ in $\mathbb{I}^{3}$. If $K=H^{2}$ on $M^{2}$, then we get

$$
\left(z_{x x}-z_{y y}\right)^{2}+4\left(z_{x y}\right)^{2}=0
$$

which yields that

$$
z_{x y}=0
$$

and

By (4.5), we derive

$$
z_{x x}=z_{y y}
$$

$$
z(x, y)=\alpha(x)+\beta(y)
$$

and considering (4.7) into (4.6) gives

$$
\frac{d^{2} \alpha}{d x^{2}}=\frac{d^{2} \beta}{d y^{2}}=d_{3}, d_{3} \in \mathbb{R} .
$$

By solving (4.8), we find

$$
\alpha(x)=\frac{d_{3}}{2} x^{2}+d_{4} x+d_{5}, \beta(y)=\frac{d_{3}}{2} y^{2}+d_{6} y+d_{7} .
$$

(4.9) implies that $M^{2}$ is either a non-isotropic plane $\left(d_{3}=0\right)$ or a parabolic sphere $\left(d_{3} \neq 0\right)$. Consequently, we have

Theorem 4.2. A graph surface of a function $z=z(x, y)$ in $\mathbb{I}^{3}$ with $K=H^{2}$ is either (a piece of) a non-isotropic plane or (a piece of) a parabolic sphere given by

$$
z(x, y)=c_{3}\left(x^{2}+y^{2}\right)+d_{8} x+d_{9} y+d_{10} .
$$

\section{References}

[1] Aydin, M.E., Ergut, M., Isotropic geometry of graph surfaces associated with product production functions in economics, Tamkang J. Math., 47(4)(2016), 433-443.

[2] Aydin, M.E., Constant curvature factorable surfaces in 3-dimensional isotropic space, arXiv:1612.02458 [math.DG].

[3] Aydin, M.E., Ogrenmis, A.O., Homothetical and translation hypersurfaces with constant curvature in the isotropic space, BSG Proceedings, 23(2016), 1-10.

[4] Bekkar, M., Senoussi, B., Factorable surfaces in the three-dimensional Euclidean and Lorentzian spaces satisfying $\triangle r_{i}=\lambda_{i} r_{i}$, J. Geom., 103(2012), 17-29.

[5] Chen, B.Y., Decu, S., Verstraelen, L., Notes on isotropic geometry of production models, Kragujevac J. Math., 38(2014), no. 1, 23-33.

[6] Chen, B.Y., Mean curvature and shape operator of isometric immersions in real-spaceforms, Glasg. Math. J., 38(1996), 87-97. 
[7] Dillen, F., Kuhnel, W., Ruled Weingarten surfaces in Minkowski 3-space, Manuscripta Math., 98(1999), 307-320.

[8] Galvez, J.A., Martinez, A., Milan, F., Linear Weingarten surfaces in $R^{3}$, Monatsh. Math., 138(2003), 133-144.

[9] Goemans, W., Van de Woestyne, I., Translation and homothetical lightlike hypersurfaces of semi-Euclidean space, Kuwait J. Sci. Eng., 38(2011), no. 2A, 35-42.

[10] Gray, A., Abbena, E., Salamon, S., Modern differential geometry of curves and surfaces with mathematica, Third Edition, Chapman and Hall/CRC Press, New York, 2006.

[11] Hou, Z.H., Ji, F., Helicoidal surfaces with $H^{2}=K$ in Minkowski 3-space, J. Math. Anal. Appl., 325(2007), 101-113.

[12] Jiu, L., Sun, H., On minimal homothetical hypersurfaces, Colloq. Math., 109(2007), 239249.

[13] Kim, M.H., Yoon, D.W., Weingarten quadric surfaces in a Euclidean 3-space, Turk. J. Math., 35(2011), 479-485.

[14] Kuhnel, W., Ruled W-surfaces, Arch. Math., 62(1994), 475-480.

[15] Lee, C.W., Linear Weingarten rotational surfaces in pseudo-Galilean 3-space, Int. J. Math. Anal., 9(50)(2015), 2469-2483.

[16] Liu, H., Liu, G., Weingarten rotation surfaces in 3-dimensional de Sitter space, J. Geom., 79(2004), 156-168.

[17] Lopez, R., Rotational linear Weingarten surfaces of hyperbolic type, Israel J. Math., 167(2008), 283-301.

[18] Lopez, R., Moruz, M., Translation and homothetical surfaces in Euclidean space with constant curvature, J. Korean Math. Soc., 52(2015), no. 3, 523-535.

[19] Meng, H., Liu, H., Factorable surfaces in Minkowski space, Bull. Korean Math. Soc., 46(2009), no. 1, 155-169.

[20] Mihai, I., On the generalized Wintgen inequality for Lagrangian submanifolds in complex space forms, Nonlinear Analysis, 95(2014), 714-720.

[21] Mihai, I., On the generalized Wintgen inequality for Legendrian submanifolds in Sasakian space forms, Tohoku J. Math, to appear.

[22] Mihai, A., Geometric inequalities for purely real submanifolds in complex space forms, Results Math., 55(2009), 457-468.

[23] Milin-Sipus, Z., Translation surfaces of constant curvatures in a simply isotropic space, Period. Math. Hung., 68(2014), 160-175.

[24] Pottmann, H., Opitz, K., Curvature analysis and visualization for functions defined on Euclidean spaces or surfaces, Comput. Aided Geom. Design, 11(1994), 655-674.

[25] Pottmann, H., Grohs, P., Mitra, N.J., Laguerre minimal surfaces, isotropic geometry and linear elasticity, Adv. Comput. Math., 31(2009), 391-419.

[26] Sachs, H., Isotrope Geometrie des Raumes, Vieweg-Verlag, Braunschweig, Wiesbaden, 1990.

[27] Strubecker, K., Differentialgeometrie des isotropen Raumes III, Flachentheorie, Math. Zeitsch., 48(1942), 369-427.

[28] Van de Woestyne, I., Minimal homothetical hypersurfaces of a semi-Euclidean space, Results Math., 27(1995), 333-342.

[29] Yu, Y., Liu, H., The factorable minimal surfaces, Proceedings of The Eleventh International Workshop on Diff. Geom., 11(2007), 33-39. 
[30] Yoon, D.W., Tuncer, Y., Karacan, M.K., Non-degenerate quadric surfaces of Weingarten type, Annales Polonici Math., 107(2013), 59-69.

Muhittin Evren Aydin

Firat University, Faculty of Science

Department of Mathematics

23200 Elazig, Turkey

e-mail: meaydin@firat.edu.tr

Alper Osman Ogrenmis

Firat University, Faculty of Science

Department of Mathematics

23200 Elazig, Turkey

e-mail: aogrenmis@firat.edu.tr 